\title{
A Virtual Exploring Robot for Adaptive Left Ventricle Contour Detection in Cardiac MR Images
}

\author{
F. Behloul, B.P.F. Lelieveldt, R.J. van der Geest, J.H.C. Reiber \\ Div. of Image Processing, Dept of Radiology, C2S, Leiden University Medical Center, \\ P.O. Box 9600, 2300 RC, Leiden, The Netherlands \\ F.Behloul@lumc.nl
}

\begin{abstract}
This paper presents an original knowledge driven automatic contour detection approach based on neuro-fuzzy techniques. The method simulates a trained virtual autonomous mobile robot that delineates the organ outlines by combining local image information and global a-priori shape knowledge. In a pilot validation study into left ventricular delineation in cardiac MR images, our novel method demonstrated a high robustness, and a clinically acceptable border localization performance.
\end{abstract}

\section{Introduction}

Automatic Left Ventricle (LV) contour detection methods encounter two major problems: (i) Manual expert contours do not always coincide with the location of the strongest local image features (the LV ENDO border is a convex hull around the blood pool), somewhat 'outside' of the strongest edge. (ii) Due to noise and image artifacts in routinely acquired clinical images, a-priori knowledge about the shape and image appearance of the LV is essential to achieve robust localization performance.

We incorporate a-priori shape and appearance knowledge and local information in the simulation and training of a virtual autonomous mobile robot that delineates fully automatically the LV.

\section{General Outline of the Method}

Our virtual robot is a tri-cycle with a steering front wheel (see Figure 1.a). The robot is subject to non-holonomic kinematic constraints: it can move only along a direction perpendicular to its rear wheel axis (continuous tangent direction) and its turning radius is lower bounded (maximum curvature). The car has a maximum curvature constraint; this provides us with an easy means to go around the papillary muscles (see figure 1.b).

The robot is provided with range sensors, mounted on its front, left and right sides. They are simulated by rays of limited length, launched from the robot in different directions. The range sensors enable the robot to estimate the distance to the myocardium borders.

The robot has to navigate around the LV cavity. To isolate the cavities from the background, a fuzzy clustering algorithm is used followed by a region growing. The gray levels are automatically grouped into 3 clusters: very bright (cavities), bright (myocardium) and dark (background). By setting the initial position of the robot outside the LV cavity and the target point inside the LV, the robot navigates around the LV cavity in a wall tracking-mode looking for an opening. Since the cavity is closed, the robot will accomplish a complete loop and stop.

W. Niessen and M. Viergever (Eds.): MICCAI 2001, LNCS 2208, pp. 1287-1288, 2001.

(C) Springer-Verlag Berlin Heidelberg 2001 
While navigating, the robot delineates the LV contours and distinguishes the septal wall (between left and right ventricular cavities) from the lateral wall. The recognition of septal and lateral wall segments is realized by sampling local image patches of the robot's environment as a grid of $3 \times 3$ regions. Each region is characterized by 3 membership degrees to the fuzzy sets: background, myocardium and cavity. The robot uses a trained fuzzy neural network to classify its local patch of image into septum or lateral. The neural network was trained on image data from 21 normal subjects.

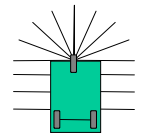

(a)

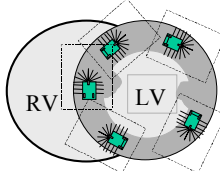

(b)

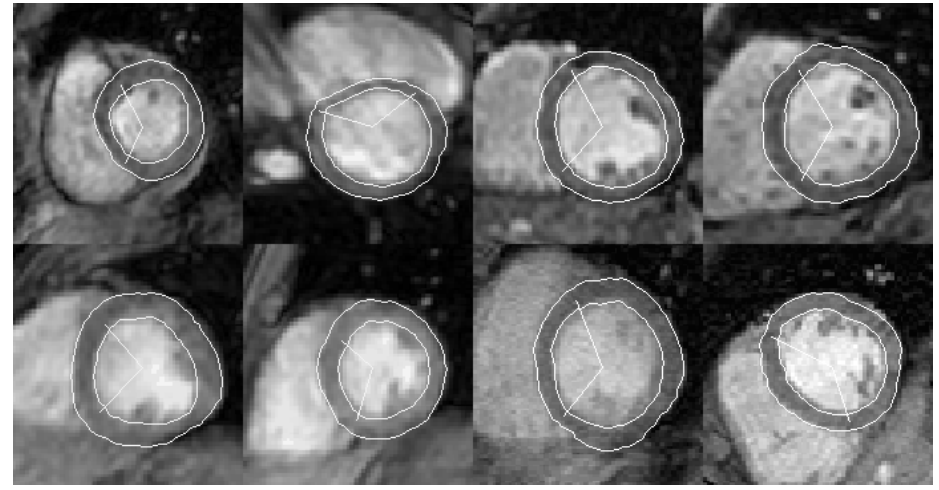

(c)

Fig. 1. (a) Tric-cycle model with range sensors (b) Navigation environment in short axis images of the heart. (c) LV contour delineation by the exploring robot. The septum is automatically delimited by the 2 lines going from the cavity to the myocardium.

\section{Results and Discussion}

The three end-diastolic mid-ventricular slices of 16 normal subjects and 7 infarct patients were considered. An expert observer was asked to draw the LV contours.

The average signed (AS) and root mean square (RMS) border positioning errors were calculated for the ENDO and EPI borders by measuring the distances between corresponding border points along 100 rays perpendicular to the centerline between the manual and the automatic contour. In addition, two clinically important area measures were calculated: ENDO area $\left(\mathrm{cm}^{2}\right)$ and EPI area $\left(\mathrm{cm}^{2}\right)$.

The automatic detected borders agreed very closely with the manually identified contours (see Figure 1.c). For the endocardium, the AS error was $-0.56 \pm 1.78 \mathrm{~mm}$ and the RMS error was $1.77 \pm 0.6 \mathrm{~mm}$. For the epicardium, the AS error was $-0.2 \pm 2.1 \mathrm{~mm}$ and the RMS error was $1.93 \pm 0.84 \mathrm{~mm}$. A good correlation was found between the observer's and the robot's LV ENDO and EPI areas $(\mathrm{y}=1.08 * \mathrm{x}-0.63, \mathrm{r}=0.89$ and $\mathrm{y}=1.02 * \mathrm{x}-0.99, \mathrm{r}=0.89)$. Average paired difference in area measures amounted to $1.1 \pm 1.84 \mathrm{~cm}^{2}$ and $0.36 \pm 2.8 \mathrm{~cm}^{2}$ for ENDO and EPI contours, respectively, indicating a very slight, but clinically acceptable, overestimation of the LV ENDO area. Our approach has been easily generalized to the image time-series from enddiastole to end-systole. It showed very high robustness to LV shape variation and deformation. 